\title{
Universal structure of mesoscale eddies in the ocean
}

\author{
Zhengguang Zhang, ${ }^{1}$ Yu Zhang, ${ }^{2}$ Wei Wang, ${ }^{1}$ and Rin Xin Huang ${ }^{3}$ \\ Received 2 May 2013; revised 8 July 2013; accepted 9 July 2013; published 30 July 2013.
}

[1] Mesoscale eddies dominate oceanic kinetic energy at sub-inertial frequencies. Their three-dimensional structure has, however, remained obscure, hindering better understanding of eddy dynamics. Here by applying the composite analysis of satellite altimetry and Argo float data to the globe, we show that despite remarkable regional differences in amplitude, extent and polarity, etc., mesoscale eddies have a universal structure in normalized stretched coordinates. Horizontally, the associated pressure anomaly is well described by a function of the normalized radial distance from the eddy center $R\left(r_{n}\right)=\left(1-r_{n}{ }^{2} / 2\right) \cdot \exp \left(-r_{n}{ }^{2} / 2\right)$, whereas vertically it is sinusoidal in a stretched coordinate $\mathrm{z}_{\mathrm{s}}=\int_{0}^{\mathrm{z}}(\mathrm{N} / \mathrm{f}) \mathrm{d} \mathrm{z}$, where $\mathrm{N}$ and $\mathrm{f}$ are the buoyancy frequency and the Coriolis parameter. Citation: Zhang, Z., Y. Zhang, W. Wang, and R. X. Huang (2013), Universal structure of mesoscale eddies in the ocean, Geophys. Res. Lett., 40, 3677-3681, doi:10.1002/grl.50736.

\section{Introduction}

[2] Mesoscale eddies are often long-lived and deepreaching vortices with horizontal scales on the order of $100 \mathrm{~km}$ in midlatitudes [Chelton et al., 2011; Roemmich and Gilson, 2001; Yang et al., 2013; Chaigneau et al., 2011]. As a ubiquitous feature in the world oceans, they are approximately in geostrophic balance, strongly constrained by the Earth's rotation and ocean stratification, affecting motions of larger and smaller scales [Farneti et al., 2010; Capet et al., 2008], and effectively transporting various tracers [Roemmich and Gilson, 2001]. Our understanding of the structure of mesoscale eddies has been rather limited due to the lack of observations. The satellite altimetry provides nearly global maps of detailed signatures of eddies at the surface [Chelton et al., 2011, 2007], but it offers no information at subsurface. Shipboard observations give measurements in the interior, but they are often very sparse and have limited depth ranges [Johannessen et al., 1989; Ledwell et al., 2008]. More recently, the composite analysis of altimetric and in situ data was adopted in a few regional studies [Roemmich and Gilson, 2001; Chaigneau et al., 2011; Liu et al., 2012; Yang et al., 2013], showing strong heterogeneity of eddy characteristics such as horizontal and vertical extents. A systematic survey of eddy structure at the global scale is still lacking, and many fundamental questions remain

\footnotetext{
${ }^{1}$ Physical Oceanography Laboratory, Ocean University of China, Qingdao, China.

${ }^{2}$ Program in Atmospheric and Oceanic Sciences, Princeton University, Princeton, New Jersey, USA.

${ }^{3}$ Department of Physical Oceanography, Woods Hole Oceanographic Institution, Woods Hole, Massachusetts, USA.

Corresponding author: Y. Zhang, Program in Atmospheric and Oceanic Sciences, Princeton University, 201 Forrestal Rd., Forrestal Campus, NJ 08540, USA. (yuz@princeton.edu)

(C)2013. American Geophysical Union. All Rights Reserved. 0094-8276/13/10.1002/grl.50736
}

unresolved. For example, what indeed do mesoscale eddies look like in three dimensions, and by what mechanisms are their structures determined? These issues not only are interesting in themselves but also pave the road for progress in our understanding of eddy dynamics.

[3] In this study, we apply the composite analysis to the globe (mostly in deep, open-ocean area due to difficulty of Argo floats in shallow, continental regions), combining surface signals of eddies detected by satellite altimetry with global coverage of hydrographic profiles from Argo floats. We will show that regional differences in the Earth's rotation (f) and ocean stratification (N) dominate the regional variation of oceanic eddy structures. When seen in stretched normalized coordinates, the pressure anomalies in association with mesoscale eddies have a universal structure worldwide; both their horizontal and vertical components can be described by simple analytical functions.

\section{Data}

[4] The altimetric data contain sea surface height (SSH) and sea surface level anomaly (SLA) products provided by Archiving, Validation, and Interpretation of Satellite Oceanographic data (AVISO). It has a weekly format covering the period from October 1992 to January 2010 on a $0.25^{\circ} \times 0.25^{\circ}$ longitude/latitude grid. The data from Argo floats consist of about 600,000 temperature and salinity $(\mathrm{T} / \mathrm{S})$ profiles in the upper $2000 \mathrm{~m}$ between years 1996 and 2010. Simple scaling of mesoscale flow fields yields a small Rossby number, implying the dominance of the geostrophic balance, so the structure of mesoscale eddies can be largely described by the field of pressure readily calculated from T/S profiles with the hydrostatic balance (see section A1).

\section{Results}

[5] Argo floats spend most of their drifting time at the parking depth (about $1000 \mathrm{~m}$ depth), and at typically 10 day intervals they quickly descend to $2000 \mathrm{~m}$ depth and then rise to the surface while measuring temperature and salinity. The distance between two neighboring profiles obtained by the same float is about $10-50 \mathrm{~km}$, relatively small compared with scales of mesoscale eddies. Indeed, almost all Argo floats observe remarkable mesoscale variations along their trajectories. More interestingly, these variations seem highly correlated at different depths. Let us take for example that the float with the platform number 1900577 drifted eastward for about 5.5 years along a semizonal path in the Southern Ocean. The curves of standardized pressure signals (see section A2) at different depths fall on top of each other (red, blue, green, and black curves in Figure 1). They are also closely aligned with a curve for the standardized surface pressure series (gray in Figure 1) constructed from the altimetric data, indicating very good vertical correlations 
Platform 1900577

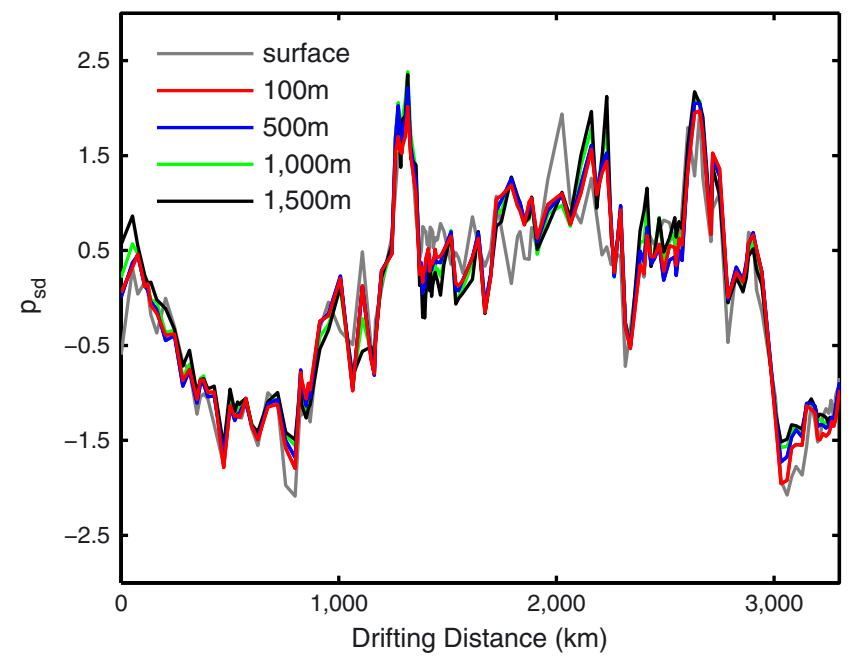

Figure 1. Standardized pressure series $\left(p_{\mathrm{sd}}\right)$ (see section A2) at different depths following the Argo float with the platform number 1900577. The standardized pressure signals are plotted at 100 (red), 500 (blue), 1000 (green), and $1500 \mathrm{~m}$ (black) depth. These curves are closely aligned and also nearly overlap with the gray curve, the standardized surface pressure series, suggesting good vertical correlations through the water column.

through the water column in the upper $2000 \mathrm{~m}$. This turns out to be a typical feature for most floats ( $70 \%$ give significantly correlated pressure series), suggesting that the horizontal structures of pressure anomalies are basically independent of depth and leading to the anticipation that eddies' horizontal and vertical structure components are separable. In other words, the pressure anomaly can be described by the product of two separable functions, i.e., $p^{\prime}=F_{1}(x, y, t) F_{2}(z)$.

[6] Mesoscale eddies were found on average axially symmetric [Chelton et al., 2011], so the field of pressure anomaly related to each eddy can be conveniently described in cylindrical coordinates $(\mathrm{r}, \mathrm{z})$, where $\mathrm{r}$ is the radial distance from the center of the eddy. The three-dimensional eddy structure is constructed in the following steps (see section A3). (1) Identify an eddy at the ocean surface using OkuboWeiss method and determine its scale, $\mathrm{R}_{0}$, and strength, $\mathrm{P}_{0}$. (2) Apply the composite analysis in a subdomain where mesoscale eddies are assumed to have the same structure. Ideally with dense-enough float profiles, any eddy detected at a given time can be grouped with a large number of profiles in its proximity, altogether determining a field of pressure anomaly $p^{\prime}(\mathrm{r}, \mathrm{z}, \mathrm{t})$. The structure of an eddy is more clearly depicted by the normalized pressure anomaly, $p_{\mathrm{n}}$, resulted from normalizing $p^{\prime}$ with $\mathrm{P}_{0}$ and normalizing its radial coordinate with $\mathrm{R}_{0}$. However in reality, there are only a limited number of float profiles at a given time, and these profiles are probably well separated in space. Therefore, we consider multiple eddies detected in a relatively small area (yet big enough to contain enough float profiles) so that all eddies, regardless of amplitude, polarity, or scale, are assumed to share the same structure. Under this assumption, pressure anomalies, which vary substantially among eddies with different $\mathrm{P}_{0}$ and $\mathrm{R}_{0}$, give the same function $p_{\mathrm{n}}$ when normalized with these two quantities. We first match each eddy with the closest float profile observed at the same time and normalize $p^{\prime}$ with $\mathrm{P}_{0}$ and $\mathrm{R}_{0}$ to get the profile of $p_{\mathrm{n}}$ at a particular normalized radial distance. Repeating this procedure for all available eddy and float profile pairs in the subdomain, we finally determine the field of $p_{\mathrm{n}}$.

[7] Applying these two steps to the globe, we have multiple fields of $p_{\mathrm{n}}$ describing eddy signals in different regions. These fields share a common feature, as anticipated from Figure 1; that is, the two variables, $\mathrm{r}_{\mathrm{n}}$ and $\mathrm{z}$, are separable, making it possible to investigate separately the radial and vertical components of the structure:

$$
p_{\mathrm{n}}\left(\mathrm{r}_{\mathrm{n}}, \mathrm{z}\right)=\mathrm{R}\left(\mathrm{r}_{\mathrm{n}}\right) \cdot \mathrm{H}(\mathrm{z})
$$

where $\mathrm{R}$ and $\mathrm{H}$ are respectively normalized functions of $r_{n}$ and $z$. Profiles of $R\left(r_{n}\right)$ identified for different regions (thin black curves in Figure 2) fall close to its average (thick gray curve), indicating the existence of a universal radial structure of pressure anomaly, as suggested by Chelton et al. [2011]. In their analysis, only the SSH field inside the core of eddies was considered, which has a single sign and varies with the radial distance in the form of a Gaussian function (their Figure 15). Unlike Chelton et al. [2011], we examined the pressure field within a much greater radial distance and found that the normalized pressure anomaly has opposite signs in and out of the core of eddies. Accordingly, the eddy has a core of single-signed anomaly of potential vorticity, surrounded by a ring of oppositely signed anomaly. Elevations of sea surface and isopycnal surfaces have the same radial structure with the pressure anomaly; their integrals over the entire range considered for an eddy tend to be zero, so the mass conservation condition for an "isolated" vortex is satisfied. Such a radial structure was observed in nature [Robinson, 1983] and in laboratory experiments [Trieling

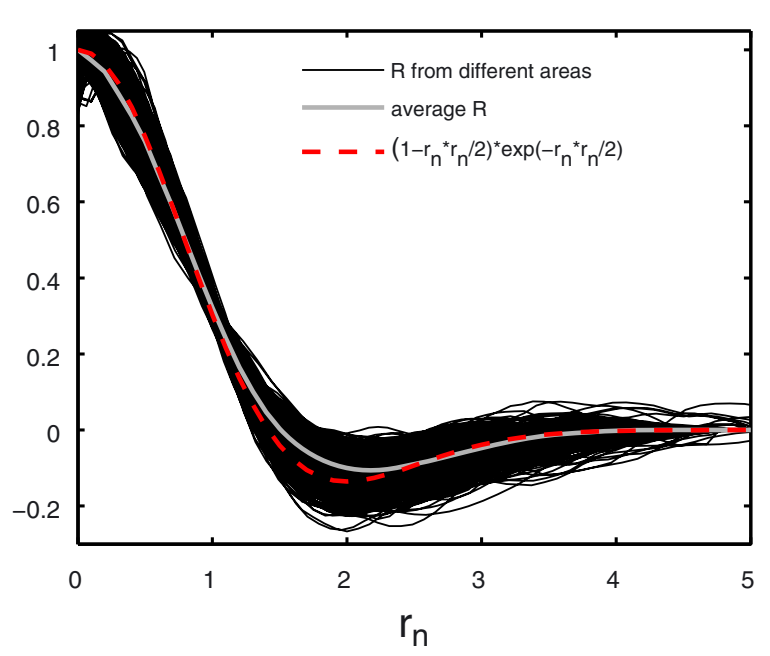

Figure 2. The universal horizontal structure of pressure in mesoscale eddies. Black curves show horizontal structures with appropriate normalization and coordinate stretching $r_{n}=r / R_{0}$ in different areas; the gray curve is their average, which is closely aligned with an analytical function $\left(1-r_{n}^{2} / 2\right) \cdot \exp \left(-r_{n}^{2} / 2\right)$ (red curve), showing the existence of a universal horizontal structure. 

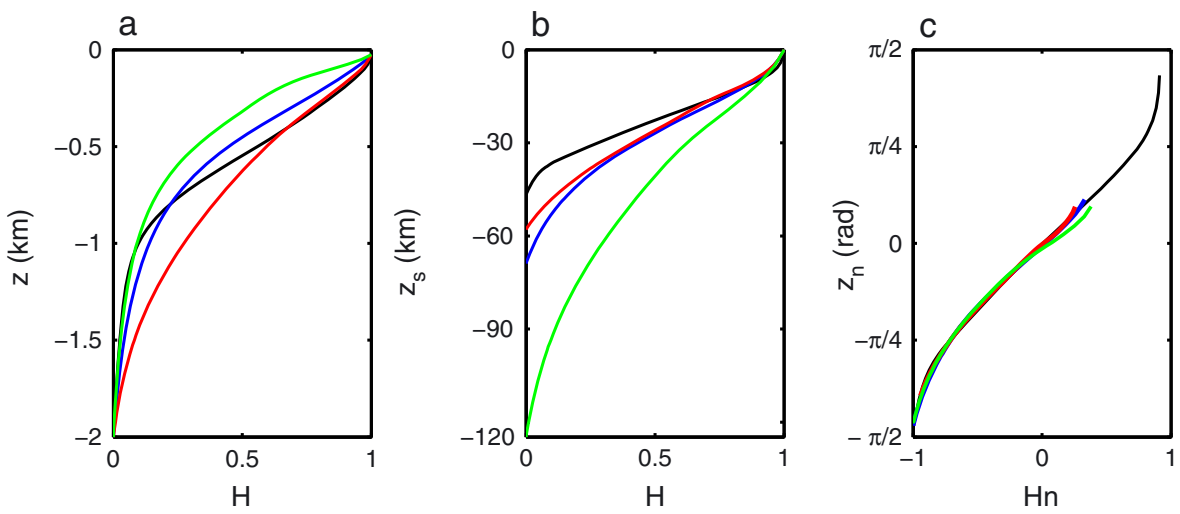

Figure 3. The common vertical structure of geostrophic eddies in four areas. (a) Vertical structures, H(z), resulting from composite analysis in regions of the Gulf Stream (black), the Kuroshio Extension (blue), the Agulhas Current (red), and the North Pacific Subtropical Countercurrent (green), showing large differences in the overall shape. (b) Vertical structures, $\mathrm{H}\left(\mathrm{z}_{\mathrm{s}}\right)$, in the stretched coordinate in those four regions, showing more or less similar shape of sine functions but with different wavelengths. (c) The normalized vertical functions, $H_{n}\left(z_{n}\right)$, are overlapping with each other.

and van Heijst, 1998; Beckers et al., 2001]. It can also be described in simple analytical form, as predicted by a theoretical model considering large-time asymptotics of potential vorticity under effects of horizontal diffusion [Kloosterziel, 1990],

$$
R\left(r_{n}\right)=\left(1-r_{n}^{2} / 2\right) \cdot \exp \left(-r_{n}^{2} / 2\right)
$$

[8] In the vertical, profiles of $\mathrm{H}(\mathrm{z})$ seem to vary substantially among different regions as shown by four examples in Figure 3a, making it difficult to discern any similar pattern out of them. However, in a stretched coordinate, $\mathrm{Z}_{\mathrm{s}}=\int_{0}^{\mathrm{z}}(\mathrm{N} / \mathrm{f}) \mathrm{dz}$, as suggested by a theoretical study of eddies within the quasi-geostrophic (QG) framework [Flierl, 1987]; they are, to the naked eye, similar in shape to sine functions, albeit different in details such as wavelength (Figure 3b). This choice of vertical coordinate was mathematically a convenient one in Flierl [1987]: it linearized the geostrophic potential vorticity and transformed the complex operator on geostrophic stream function to a three-dimensional Laplacian with an extra term, which is relatively small in general. In the present study, the application of such a coordinate, $z_{S}$, is essential for discovery of the universal vertical structure. We fit $\mathrm{H}\left(\mathrm{z}_{\mathrm{S}}\right)$ to a sine function plus a constant value,

$$
\mathrm{H}_{0} \cdot \sin \left(\mathrm{kz}_{\mathrm{s}}+\theta_{0}\right)+\mathrm{H}_{\mathrm{ave}}
$$

and take parameters $\mathrm{H}_{0}, \mathrm{k}, \theta_{0}$, and $\mathrm{H}_{\mathrm{ave}}$ as unknowns to be determined. Amazingly, curves for those four examples, when normalized by the fitting results, $\mathrm{H}_{\mathrm{n}}=\left(\mathrm{H}\left(\mathrm{z}_{\mathrm{s}}\right)-\right.$ $\left.\mathrm{H}_{\text {ave }}\right) / \mathrm{H}_{0}$, and plotted against a new coordinate, $\mathrm{z}_{\mathrm{n}}=\mathrm{kz}_{\mathrm{s}}+$ $\theta_{0}$, collapse onto a single curve (Figure 3c). Repeating these stretching and fitting procedures in all areas leads to Figure 4, where all curves are closely aligned to their average (thick gray curve), which overlaps with that of the analytical function, $\sin \left(z_{n}\right)$ (thick red curve). This vertical sine structure, with only one zero-crossing in the interior and vanishing derivative at the lower boundary, is somewhat similar to the classical, free, first baroclinic mode [Gill, 1982; Wunsch, 1997] but is meanwhile distinguished by one important feature. Its phase, $\theta_{0}$, is nonzero at the surface and varies markedly among regions, presumably resulting from differential surface dynamics. The classical, first, baroclinic mode, on the other hand, is one of the normal modes arising from the QG equations that are linearized about a mean state with an interior potential vorticity gradient on a flat bottom but have no surface buoyancy perturbations. This being said, it seems sensible to regard the sine structure $\sin \left(z_{n}\right)$ as a special kind of first baroclinic "mode" taking into account contributions of surface buoyancy anomaly. The fact that the vertical structure as shown in equation (3) is dominated by the barotropic and the first baroclinic mode is consistent with the suggestion of an observational study that the eddy kinetic energy in the ocean primarily resides in the barotropic and first baroclinic modes [Wunsch, 1997].

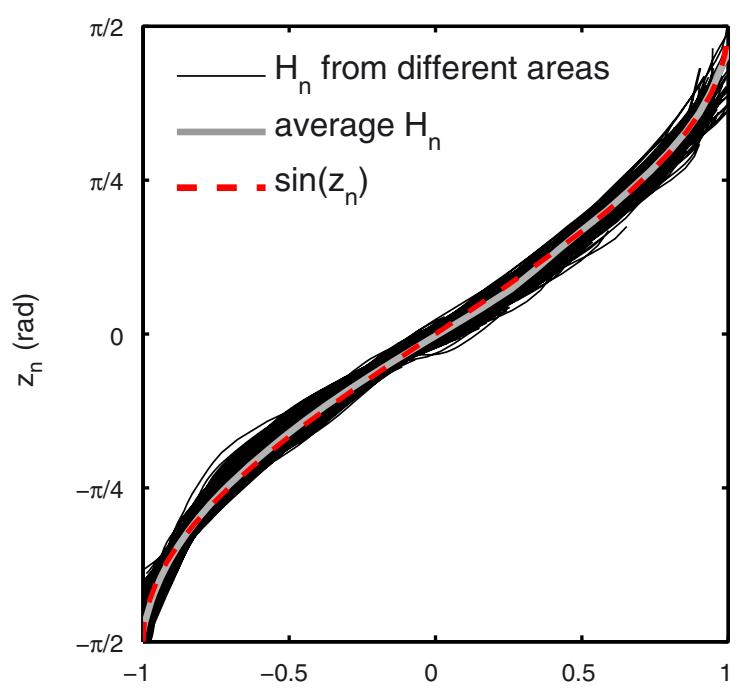

Figure 4. The universal vertical structure of mesoscale eddies. Black curves show the normalized vertical function, $\mathrm{H}_{\mathrm{n}}\left(\mathrm{z}_{\mathrm{n}}\right)$ for all areas; the gray curve depicts their average, which is closely aligned with the analytical function $\sin \left(z_{n}\right)$ (red curve), showing the existence of a universal vertical structure. 


\section{Conclusion}

[9] Based on available altimetric and profiling float data, our composite analysis of the three-dimensional eddy structure reveals an important common feature of mesoscale eddies in the world oceans. They have horizontal and vertical structure components that can be described separately, and both components are universal in normalized stretched coordinates. The universal shape in the vertical is not perceptible until the coordinate is stretched with the factor $\mathrm{N} / \mathrm{f}$, demonstrating the key role of the Earth's rotation and ocean stratification in causing regional differences of eddies' structures. It should be noted that in this new coordinate, different depth ranges of the ocean are disproportionally "stretched" depending on their stratification. Consequently, signals get emphasized as seen under a magnifier in the main thermocline where stratification is strong, but those in the surface mixed layer and in the vast expanse of the deep ocean with very weak stratification are compressed.

[10] There are two assumptions considered in this study, the first one is hydrostatic balance, used in calculation of pressure from density profile, and the second one is geostrophic balance, which is applied in detection of eddies with the Okubo-Weiss method and is not indispensable for our analysis. For example, if the SSH-based method is used to detect eddies, as in Chelton et al. [2011], there is no need to assume geostrophic balance. Therefore, other than the hydrostatic balance, both the composite analysis and the universal eddy structure are based solely on observations, free of any dynamical assumptions.

[11] Unraveling the three-dimensional structure of eddies in the ocean is a grand challenge. As the first attempt to investigate mesoscale eddies at the global scale, the current study takes full advantage of the two global measurements. It is focused on identifying the global universal structure at the lowest order by exploring similarities rather than emphasizing differences over different regions. Such a universal structure may provide a useful tool in examining the role of eddies in the oceanic circulation and climate. Further study in refining this structure is expected, and the refined structure can serve as a benchmark for numerical models where mesoscale eddies are explicitly resolved. In addition, the generation mechanism for this universal structure remains unknown; thus, exploring such mechanism may bring new excitement to eddy research.

\section{Appendix: Methods}

\section{A1. Computing the Pressure Profile From a Given T/S Profile}

[12] Potential density, $\rho_{\theta}$, referenced to the sea surface is directly computed from the T/S profile. Integrating upward the equation of the hydrostatic balance from a given reference level (here $2000 \mathrm{~m}$ depth is chosen for convenience) leads to

$$
p(\mathrm{x}, \mathrm{y}, \mathrm{z}, \mathrm{t})=-\int_{-2,000}^{\mathrm{z}} \mathrm{g} \rho_{\theta}\left(\mathrm{x}, \mathrm{y}, \mathrm{z}^{\prime}, \mathrm{t}\right) \mathrm{dz} \mathrm{z}^{\prime}+\mathrm{C},
$$

where $\mathrm{g}$ is the gravitational acceleration and $\mathrm{C}$ is the pressure at $2000 \mathrm{~m}$ depth. It should be noted that choosing a different reference level will change the value of $\mathrm{C}$ but will not affect eddy structures resulting from composite analysis.

\section{A2. Computing Pressure Anomalies at Different Depths on the Path of a Given Argo Float}

[13] On the drifting path of the Argo float, we have a series of $p(\mathrm{x}, \mathrm{y}, \mathrm{z}, \mathrm{t})$ at different depths $(\mathrm{z}<0)$ inferred from float data and a series of surface pressure, $p(\mathrm{x}, \mathrm{y}, 0, \mathrm{t})$, computed from altimetry:

$$
p(\mathrm{x}, \mathrm{y}, 0, \mathrm{t})=\mathrm{g} \rho_{0} \mathrm{SSH},
$$

where $\rho_{0}$ in equation (A2) is the density at the surface, set to the background mean density $1030 \mathrm{~kg} \mathrm{~m}^{-3}$. Using the standardization method for time series, including removal of the mean value and normalization with standard deviation, we get the standardized pressure signals, $p_{\text {sd }}(\mathrm{x}, \mathrm{y}, \mathrm{z}, \mathrm{t})$, at and below the surface, as plotted in Figure 1.

\section{A3. Constructing a Three-Dimensional Pressure Field in a Subdomain With the Radius of $15^{\circ}$}

[14] We divide the entire globe into $5^{\circ} \times 5^{\circ}$ grid cells, each of which is the center of a subdomain, a circular area with the radius of $15^{\circ}$. This circular area is thought to be qualified for the regional composite analysis if it contains more than 5000 Argo float profiles.

[15] Step 1: Data preparation. Having obtained the pressure signals for all $\mathrm{T} / \mathrm{S}$ profiles, we take their multiyear seasonal average and regard it as a "climatology", which is subtracted from the original signal to give the pressure anomalies $p^{\prime}$ for the globe.

[16] Step 2: Eddy detection. We first search, within the subdomain, for local maximum (minimum) of SLA as centers of possible anticyclones (cyclones). To further determine whether these eddies are real, we make use of the Okubo-Weiss criterion [Isern-Fontanet et al., 2003]: an eddy is confirmed if its center is embedded within a closed contour of the Okubo-Weiss parameter $\mathrm{W}=-2 \times 10^{-12} \mathrm{~s}^{-2}$. This parameter is a measure of the relative importance of rotation and deformation in fluid flow, defined as

$$
\mathrm{W}=4\left(\mathrm{u}_{\mathrm{x}}^{2}+\mathrm{v}_{\mathrm{x}} \mathrm{u}_{\mathrm{y}}\right)
$$

for flow without horizontal divergence as in geostrophic balance, where velocity components are computed from SLA by geostrophic relation:

$$
\begin{aligned}
& u=-\mathrm{gf}^{-1} \frac{\partial(\text { SLA })}{\partial y}, \\
& \mathrm{v}=\mathrm{gf}^{-1} \frac{\partial(\text { SLA })}{\partial \mathrm{x}} .
\end{aligned}
$$

The area, A, enclosed by this particular $\mathrm{W}$ contour sets the scale of the eddy by $R_{0}=\sqrt{A / \pi}$. The strength of the eddy is defined by the surface pressure anomaly at the eddy center, $\mathrm{P}_{0}=\mathrm{g} \rho_{0}$ (SLA) $)_{\text {center }}$. To avoid contamination of nearby eddies of opposite polarity, we excluded eddies that have centers of other eddies appear within the normalized radial distance 3.

[17] Step 3: Composite analysis. Then, each pressure anomaly profile, $p^{\prime}$, in the subdomain is matched with the closest surface-detected eddy occurring at the same time (considered as "simultaneous" since the maximum difference of occurring time between the Argo profile and the "closest" eddy detected on the atlimetric map is less than 3.5 days, short compared with the typical life span of 


\section{ZHANG ET AL.: STRUCTURE OF OCEANIC GEOSTROPHIC EDDIES}

mesoscale eddies). Normalizing $p^{\prime}$ with the eddy strength $\mathrm{P}_{0}$ and its radial coordinate with the eddy scale $\mathrm{R}_{0}$, we have

$$
p_{\mathrm{n}}\left(\mathrm{r}_{\mathrm{n}}, \mathrm{z}, \mathrm{t}\right)=p^{\prime}\left(\mathrm{r} / \mathrm{R}_{0}, \mathrm{z}, \mathrm{t}\right) / \mathrm{P}_{0} .
$$

Assuming eddies within this local area have the same structure regardless of strength or scale, we thus construct a single field of pressure anomaly $p_{\mathrm{n}}\left(\mathrm{r}_{\mathrm{n}}, \mathrm{z}\right)$ for a given area. Repeating steps 2 and 3 leads to multiple $p_{n}\left(r_{n}, z\right)$ that share a universal structure in the normalized stretched coordinates.

[18] Acknowledgments. The authors thank China Argo Real-time Data Center for providing their Argo floats data. This research was supported by the National Natural Science Foundation of China under grant 41276014 and the National Basic Research Priorities Program of China through grant 2013CB430303. The authors thank Dudley B. Chelton and another anonymous reviewer for their constructive comments, which improved the quality of the article.

[19] The Editor thanks Dudley Chelton and an anonymous reviewer for their assistance in evaluating this paper.

\section{References}

Beckers, M., R. Verzicco, H. Clercx, and G. van Heijst (2001), Dynamics of pancake-like vortices in a stratified fluid: Experiments, model and numerical simulations, J. Fluid. Mech., 433, 1-27.

Capet, X., J. McWilliams, M. Molemaker, and A. Shchepetkin (2008), Mesoscale to submesoscale transition in the California Current system. Part I: Flow structure, eddy flux, and observation tests, J. Phys. Oceanogr., 38, 29-43.

Chaigneau, A., M. Texier, G. Eldin, C. Grados, and O. Pizarro (2011), Vertical structure of mesoscale eddies in the eastern South Pacific Ocean: A composite analysis from altimetry and Argo profiling floats, J. Geophys. Res., 116, C11025, doi:10.1029/2011JC007134.

Chelton, D., M. Schlax, and R. Samelson (2011), Global observations of nonlinear mesoscale eddies, Prog. Oceanogr., 91, 167-216.
Chelton, D., M. Schlax, R. Samelson, and R. de Szoeke (2007), Global observations of large oceanic eddies, Geophys. Res. Lett., 34, L15606, doi:10.1029/2007GL030812.

Farneti, R., T. Delworth, A. Rosati, S. Griffies, and F. Zeng (2010), The role of mesoscale eddies in the rectification of the Southern Ocean response to climate change, J. Phys. Oceanogr., 40, 1539-1557.

Flierl, G. R. (1987), Isolated eddy models in geophysics, Ann. Rev. Fluid Mech., 19, 493-530.

Gill, A. E. (1982), Atmosphere-Ocean Dynamics, 664 pp., Academic Press, New York.

Isern-Fontanet, J., E. García-Ladona, and J. Font (2003), Identification of marine eddies from altimetric maps, J. Atmos. Oceanic Technol., 20, $772-778$.

Johannessen, J. A., S. Sandven, K. Lygre, E. Svendsen, and O. Johannessen (1989), Three-dimensional structure of mesoscale eddies in the Norwegian Coastal Current, J. Phys. Oceanogr., 19, 3-19.

Kloosterziel, R. C. (1990), On the large-time asymptotics of the diffusion equation of infinite domains, J. Eng. Math., 24, 213-236.

Ledwell, J. R., D. McGillicuddy Jr., and L. Anderson (2008), Nutrient flux into an intense deep chlorophyll layer in a mode-water eddy, Deep-Sea Res. II., 55, 1139-1160.

Liu, Y, C. Dong, Y. Guan, D. Chen, J. McWilliams, and F. Nencioli (2012), Eddy analysis in the subtropical zonal band of the North Pacific Ocean, Deep-Sea Res. I., 68, 54-67.

Robinson, A. R. (1983), Eddies in Marine Science, 644 pp., Springer, Berlin.

Roemmich, D., and J. Gilson (2001), Eddy transport of heat and thermocline waters in the North Pacific: A key to interannual/decadal climate variability? J. Phys. Oceanogr., 31, 675-687.

Trieling, R. R., and G. van Heijst (1998), Decay of monopolar vortices in a stratified fluid, Dyn. Res., 23, 27-43.

Wunsch, C. (1997), The vertical partition of oceanic horizontal kinetic energy, J. Phys. Oceanogr., 27, 1770-1794.

Yang, G., F. Wang, Y. Li, and P. Lin (2013), Mesoscale eddies in the northwestern subtropical Pacific Ocean: Statistical characteristics and three-dimensional structures, J. Geophys. Res. Oceans, 118, 1906-1925, doi:10.1002/jgrc.20164. 